\title{
A NOTE \\ ON PRONUNCIATION
}

The introduction and translations are larded with names of Chinese people, places, and things. The pinyin system of spelling Chinese has been used throughout. Some Chinese words are easily pronounced, others are not. This note is primarily intended to help with the latter. Although not alphabetic, the arrangement of names possesses some modicum of logic. In the course of this note, I often mention the Yale system of romanization because, while it is not as accurate as pinyin, it is sometimes easier for speakers of English to pronounce.

\section{Abbreviations}

MBA may be (roughly) approximated

Cw Chinese word

EW English word

RW rhymes with

SL sounds like

Any word in quotation marks following either RW or SL is assumed to be English. For example, mang Rw gong means that the Chinese word "mang" rhymes with the English word "gong."

Cai Yuanpei INTRODUCTION

Yuan Shikai PREFACE
Let us begin with Cai Yuanpei. The ai in Cai RW high. Easy enough. The $c$ sound, however, does not exist in English. Cai MBA by saying, $I t$ 's $I$, and then dropping the initial $I$. (The Yale system spells this sound tsai.)

As with all Chinese names, the family name appears first, the given name second. Yuan MBA by saying You Anne rapidly enough to elide the two words; pei SL pay.

Yuan as in the preceding entry. Shi MBA by saying Ew her and then putting an $s$ in front, sher-now take the vowel out and you will 
have something like $\mathrm{Cw}$ Shi. (Yale spells it Shr.) Kai RW sky.

Master Yangsheng Yang RW gong; sheng Rw sung. REMEMBRANCE

While we are on names, how does one know where to break a given name into its typical two syllables? For the most part, this will be obvious; if it is not, remember that all Mandarin syllables end in a vowel, diphthong, $n$, $n g$, or-rarely-an $r$. Sometimes when the break is not apparent, pinyin indicates it with an apostrophe. Let us take some examples.

Hong'er HOMETOWN

Kong Yiji KONG YIJI
The Hong in Hong'er is a sound which is rare in English, but easy to make. The $o$ SL the oo of Ew hoot. Substitute $n g$ for the $t$ in hoot and you will have something like $\mathrm{Cw}$ hong. Perhaps some readers will remember the famous Soong sisters and their brother, T. V. Soong. Hong RW their surname (spelled Song in pinyin / Sung in Yale). The er is easy enough - SL the American pronunciation of EW are.

While we are on the Chinese ong sound, it would be well to deal with this protagonist of the story by the same name. The Kong is very close to EW coot, with an $n g$ substituted for the final $t$. The Yi sL the $e$ in EW she; the $j i \mathrm{SL}$ gee as in gee whiz! Now back to the apostrophe.

Xiu'er SOAP
The Xiu presents us with the strange-looking initial $x$. For the purposes of rough approximation the English $s$ sound will do. The iu part MBA by saying yo in Ew yo-yo. Put an $s$ before the yo, and you have something similar to $\mathrm{Cw}$ xiu.

Quite a few $x$ initials occur in the introduction and translations, both in names of real people: Xu Guangping, Xu Qinwen, Xu 
Shoushang, Xu Xilin; and in names of fictional characters: Xia Yu, Sister Xianglin, and Xuecheng. Let us examine a few of the more difficult ones.

Xu Qinwen

HAPPY FAMILY

Xu Guangping INTRODUCTION

Xu Shoushang PREFACE

Sister Xianglin NEW YEAR'S

huixiang dou MEDICINE

Kangxi NEW YEAR'S

mantou MEDICINE

Fan Ainong INTRODUCTION
We do not have the $u$ of $X u$ in English, although Ew you will give a very rough approximation. Thus, $X u$ is like see you said rapidly enough to drop the ee from see. Qinwen MBA by saying Ews chin one.

Lu Xun's second wife was also surnamed $X u$. The Guang of her given name MBA by saying CW wang (RW gong) and then tacking a $g$ on the front: gwang. (And the Yale system would spell it so.) CW ping SL ping in EW ping-pong.

Shou SL show; shang RW gong.

$X i$ in Xiang MBA by Ew see; the ang of Xiang RW gong; lin SL lean.

Hui MBA by saying EW way and then putting an $h$ in front of it; xiang as in the preceding entry; dou SL doe.

Kang RW gong; $x i$ MBA by EW see.

Man MBA by saying EW man (the an, however, is as close to EW on as it is to the an of EW man); tou SL toe.

Fan MBA by saying Ew fan (as in the preceding entry, however, the an sound is also close to EW on); Ai SL I; for nong drop the se from EW noose and replace with $n g$. (Yale spells it nung.) 
$x \mid v i$

Xuecheng SOAP

Lu Xun

Zhao'er SOAP

Zhuang Qiguang ETERNAL LAMP

zha

HOMETOWN

Zhang Xun INTRODUCTION

The Xue is a bit tricky. Say Ew yeah (yes), then put a $w$ between the $y$ and $e a b$ and say $y w e a h$. This is roughly equivalent to the $u e$ in cw Xue. Now put an $s$ up front, say syweah, and you have something like $\mathrm{cw}$ Xue. $\mathrm{cw}$ cheng RW sung.

While still on the $x$ sound, let us take up the pen name of the author, Lu Xun (his real name was Zhou Shuren-Zhou SL Joe; Shu SL shoe; and ren SL wren). The $L u \mathrm{RW} d o$ and the $X u n$ sL see you said rapidly, dropping the ee in see and then tacking an $n$ on the end. (Yale spells this syun.)

The $z h$ SL the $j$ in EW jowl; the ao RW the ow in EW how. (Pinyin would spell Ew how as hao.) For er see the entry on Xiu'er above.

First say wang for the uang (the $u$, after all, represents a $w$ sound) so as to rhyme with $\mathrm{EW}$ bong, then put a $j$ in front of it (for the $z h$ ) and you will end up with something like $\mathrm{Cw}$ Zhuang. Q $i$ is like the chee in $\mathrm{EW}$ cheer; guang should pose no problem, just put a $g$ in front of the same uang sound that occurs in $\mathrm{CW}$ Zhuang and you will have something like $\mathrm{cw}$ guang. (Yale spells this one gwang.)

The name of this animal is quite easy. RW $p a$ or $m a ; z b$ sL $j$. (Yale spells it $j a$.)

Again the ang RW gong; the $z h$ SL $j$, giving us something which SL the surname of the American author Erica Jong. For Xun, see Lu Xun above. 
Zhang Peijun BROTHERS

Zhao Sichen Zhao Baiyan AH Q

Zhejiang

Zijun MOURNING

Juansheng MOURNING

Siming SOAP

Zhou Zuoren INTRODUCTION
Zhang as above; Pei sL pay, and jun MBA by saying $\mathrm{EW}$ June.

See Zhao'er above for Zhao and Siming below for si. The $c h$ of CW chen SL the $c h$ of Ew church; chen $\mathbf{R W}$ ton. Bai SL buy, and yan SL yen.

This is Lu Xun's native province. The Zhe SL the $\boldsymbol{u} \boldsymbol{b}$ of the English expression $u h-b u b$ with an English $j$ tacked on front: jub; jiang sL the gee of the English expression gee whiz! with the ong of EW gong tacked on the end (geeong) and said quickly as a single syllable.

$Z i$ is a bit tricky; it MBA by saying the $d s$ in EW suds or buds (the result sounds like $d z$, the spelling Yale uses); Ew June will give you a very rough, but acceptable, version of $\mathrm{cw} j u n$.

For Juansheng, the name of Zijun's lover, go all the way back to the pronunciation of Cai Yuanpei's name at the beginning of this note. Follow the directions for yuan and then put a $j$ in front to make cw Juan (Yale spells jywan); the second syllable, sheng, RW sung.

The $S i$ in Siming is not pronounced like the English sigh, but very roughly like the $s u$ of EW suds; if you can make the $u$ sound like a English $z$, you will be even closer (Yale spells it $s z$ ); ming rhymes with $\mathrm{EW}$ sing.

The name of Lu Xun's younger brother appears quite often in the introduction and notes to the stories. The Zhou can be approximated, as noted above, by simply saying $\mathbf{E w}$ Joe. (The same name as China's famous premier, Zhou Enlai.) Zuoren presents some difficulties. The $z$ is the familiar $d s$ of EW buds 
Zhou Jianren INTRODUCTION

Fang Xuanchuo DRAGONBOAT

Kuoting ETERNAL LAMP

Qian Xuantong INTRODUCTION

Qiu Jin INTRODUCTION

Lü Weifu UPSTAIRS while the uo MBA by saying EW awe and then tacking a $w$ on front-wawe. Now say dswawe and you will have something like $\mathrm{Cw}$ Zuo; ren SL wren.

This was the youngest of the three Zhou brothers. Jian MBA by saying EW jenny and then dropping the $y$. Note that all three brothers have ren as the last syllable of the given name. This serves as a generational marker.

Fang RW gong. Xuan MBA by saying See you, Anne fast enough to fuse the words into a single syllable; chuo consists of the same uo encountered in Zhou Zuoren's name above (pronounced wawe-EW awe with a $w$ tacked on the front) with the $c h$ in EW church put in front of it: chwawe.

The $u o$ in Kuo MBA by saying wawe as in the previous entry; tack a $k$ on the front and you have kwawe, which roughly approximates the sound of $\mathrm{cw}$ Kuo. Ting Rw sing.

Qian SL yen with a $c h$ as in EW cheer tacked on the front: chyen; Xuan as in the entry for Fang Xuanchuo above; tong MBA by saying EW toot while substituting an $n g$ for the final $t$.

For Qiu say the chee of Ew cheer and then add the $o$ of EW go; Jin SL gin.

The $L \ddot{u}$ may be approximated by putting an $l$ in front of EW you. Note this is not the same sound as the $L u$ in Lu Xun. The pronunciation of Weifu is obvious.

$\mathrm{He}$ stories. It is not pronounced like the English 
third person pronoun. The $e$ comes from deep in the throat so that $\mathrm{CW} H e$ sounds like $\mathrm{EW}$ buh in Huh, what's that you said?

He Goubao

REMEMBRANCE

Yaozong

REMEMBRANCE
Gou SL go; bao Rw how.

Yao RW how; zong is difficult. Say the $d s$ of EW suds then tack an ong on it. (The pronunciation of ong is explained in the Kong Yiji entry above.)

Seventh Sister Zou For the Zou, say the $d s$ in EW suds and then AH Q tack EW owe onto it: dsowe.

Mother Liu NEW YEAR'S

Runtu HOMETOWN

Wei Lianshu LONER
Liu is included because it is so often mispronounced. It does not SL EWs Lee (surname) and you said together, but more like Ew Leo said as a single syllable.

Going back to "Hometown" for a moment, let us take up one of the most difficult names. The $t u$ in Runtu presents no problem. Say Ew two and you will be close enough. For the $R u n$, say EW wood, then replace the $d$ with an $n$. Put an $r$ in front of that and you will have something like CW Run. It is not an easy sound to make.

Here is an easy one. Wei is just like Ew way. $S h u$ is just like the shoe that you wear. For Lian, take the English name Lee and tack EW yen on the end of it; now say the two words fast enough to elide them into a single syllable and you will have something like CW Lian; shu sounds just like shoe (footwear). 
Gao Erchu VENERABLE

Aigu

DIVORCE
Gao RW how. Er is like the American-English pronunciation of are; chu is like $\mathrm{EW}$ chew.

Ai SL I; gu SL goo.

For more accurate pronunciations than any of those given above, go to a good textbook on learning Mandarin or to a Chinese friend. 


\section{Diary of a Madman and Other Stories}


